\title{
GROUP RINGS OVER FROBENIUS RINGS
}

\section{SAMIR ASSUENA}

Centro Universitário da FEI

Av. Humberto de Alencar Castelo Branco 3972

São Bernardo do Campo-SP

Brazil

e-mail: samir.assuena@fei.edu.br

sassuena@yahoo.com.br

\begin{abstract}
In this paper, we consider group rings $R G$ of finite groups over Frobenius rings. We introduce the concept of Jacobson ring for $G$ and give necessary conditions over $R$ to $R G$ be a Frobenius ring.
\end{abstract}

\section{Introduction}

A ring $R$ is called quasi-Frobenius ( $Q F$ ring for short) if $R$ is right noetherian and right self-injective. The class of $Q F$ rings appeared for first time in the work of Brauer, Nesbitt, Nakayama, and others, in the form of Frobenius algebras. The study of such algebras was motivated by the representation theory of finite groups. Since then, $Q F$ rings have been studied and have been also used in coding theory. For instance, Wood proved that a finite ring $R$ has the extension property for Hamming weight if and only if $R$ is Frobenius ([5], Theorems 2.2 and 2.3). 
We say that a $Q F$ ring $R$ is a Frobenius ring if $\operatorname{soc}\left({ }_{R} R\right) \cong_{R}(R / J(R))$ as $R$-modules and for $Q F$ rings, we have an important and very interesting result, due to Nakayama ([3]), which shows that $R$ is $Q F$ if and only if $G$ is finite and $R G$ is $Q F$.

Since group algebras $\mathbb{F} G$ of finite groups $G$ over a field $\mathbb{F}$ which are finite-dimensional are Frobenius rings ([1], Example 16.56 and Theorem 16.21) and if $R$ is a finite Frobenius ring and $G$ is a finite group, then the group ring $R G$ is also Frobenius ([4], Example $4.4(\mathrm{v})$ ), we are interested in studying the following equivalence: $R$ is Frobenius if and only if $R G$ is Frobenius.

In this paper, we shall show that this equivalence holds for a class of rings $R$.

\section{Basic Results}

In this section, we shall present basic results which will be used in this paper.

Let $R$ be a ring. In this paper, $J(R)$ will always denote the Jacobson radical of $R$ and $\operatorname{soc}\left(R_{R}\right)$ and $\operatorname{soc}\left({ }_{R} R\right)$ the right and left socle of $R$.

Lemma 2.1. For any artinian ring $R$, we have:

$\operatorname{soc}\left({ }_{R} R\right)=\{r \in R \mid J(R) \cdot r=0\}$ and $\operatorname{soc}\left(R_{R}\right)=\{r \in R \mid r \cdot J(R)=0\}$.

Theorem 2.2 ([2], Theorem 2.7.16). Let $R$ be a semisimple ring. Then, $R$ is artinian and the following conditions hold:

(i) $R$ contains no nonzero nilpotent two-sided ideals;

(ii) $R$ contains no nonzero nilpotent left ideals;

(iii) $J(R)=(0)$.

Conversely, if $R$ is artinian and any of the above conditions holds, then $R$ is semisimple. 
Theorem 2.3 ([1], Theorem 15.1). Let $R$ be a ring. Then, $R$ is $Q F$ if and only if $R$ is (2-sided) artinian and the following conditions hold:

(1) $\operatorname{ann}_{r}\left(\operatorname{ann}_{l}(A)\right)=A$ for any right ideal $A \subseteq R$;

(2) $\operatorname{ann}_{l}\left(\operatorname{ann}_{r}(B)\right)=B$ for any left ideal $B \subseteq R$.

Proposition 2.4 ([1], Corollary 15.6). For any $Q F$ ring $R$, we have

$$
\operatorname{ann}_{l}(J(R))=\operatorname{soc}\left(R_{R}\right)=\operatorname{soc}\left({ }_{R} R\right)=\operatorname{ann}_{r}(J(R)) .
$$

Proposition 2.5 ([1], Example 15.6-3). Let $R=\prod_{i=1}^{n} R_{i}$. Then $R$ is $Q F$ ring if and only if $R_{i}$ is $Q F$, for all index $i$.

Proposition 2.6 ([3]). Let $R$ be a ring and $G$ be a finite group. Then $R$ is $Q F$ if and only if $R G$ is $Q F$.

Theorem 2.7 ([1], Theorem 15.27). For any commutative ring $R$, the following are equivalent:

(1) $R$ is $Q F$;

(2) $R \cong R_{1} \times R_{2} \times \cdots \times R_{s}$, where each $R_{i}$ is a local artinian ring with simple socle.

Definition 2.8. Let $R$ be a $Q F$ ring. We say that $R$ is a Frobenius ring if $\operatorname{soc}\left({ }_{R} R\right) \cong_{R}(R / J(R))$ as $R$-modules.

Lemma 2.9 ([4], Remark 1.3). Let $R$ be a commutative artinian ring. Then $R$ is $Q F$ if and only if $R$ is Frobenius.

Proposition 2.10 ([1], Example 16.19-3). Let $R=\prod_{i=1}^{n} R_{i}$. Then $R$ is Frobenius ring if and only if $R_{i}$ is Frobenius, for all index $i$.

It follows from Proposition 2.6 and Lemma 2.9 that if $R$ is commutative ring, $G$ finite group and if $R G$ is Frobenius, then $R$ is Frobenius. Wood proved the converse of this result for finite Frobenius rings. 
Proposition 2.11 ([4], Example 4.4 (v)). Let $R$ be a finite Frobenius ring and $G$ be a finite group. Then $R G$ is Frobenius.

Theorem 2.12 (Maschke's Theorem [2], Theorem 3.4.7). Let $G$ be a group and $R$ be a ring. Then, the group ring $R G$ is semisimple if and only if the following conditions hold:

(i) $R$ is a semisimple ring;

(ii) $G$ is finite;

(iii) $|G|$ is invertible in $R$.

\section{New Results}

Let $R$ be an artinian ring, $G$ be a finite group, and $R G$ be the group ring of $G$ over $R$. We denote by $J(R)$ the Jacobson radical of $R$ and by $J(R G)$ the Jacobson radical of $R G$.

Since $R$ is artinian ring, the ideal $J(R)$ is nilpotent ideal and, it is not difficult to see, the set

$$
J(R) G=\left\{\sum_{g \in G} a_{g} g \mid a_{g} \in J(R)\right\}
$$

is an ideal of $R G$ and $R G / J(R) G \cong(R / J(R)) G$. Since $J(R)$ is nilpotent, the ideal $J(R) G$ is also nilpotent so $J(R) G \subseteq J(R G)$.

Definition 3.1. Given a finite group $G$, we say that an artinian ring $R$ is a Jacobson ring for $G$ if the equality $J(R) G=J(R G)$ holds.

Proposition 3.2. Given a finite group $G$, an artinian ring $R$ is a Jacobson ring for $G$ if and only if $|G| \in \mathcal{U}(R / J(R))$. 
Proof. Suppose that $|G| \in \mathcal{U}(R / J(R))$. Since $R$ is artinian, the factor ring $R / J(R)$ is semisimple ring, so $(R / J(R)) G$ is also semisimple by hypothesis. Thus, $J((R / J(R)) G)=0$. Consequently, $J(R G / J(R) G)=0$ and the equality $J(R) G=J(R G)$ holds.

Now, suppose that the equality $J(R) G=J(R G)$ holds. Since $J((R / J(R)) G)=J(R G / J(R) G)=0$ and $(R / J(R)) G$ is artinian, the group ring $(R / J(R)) G$ is semisimple, then, by Maschke's theorem, $|G| \in \mathcal{U}(R / J(R))$.

Corollary 3.3. Let $R$ be an artinian local ring and $G$ be a finite group such that $m=\operatorname{char}(R)$ does not divide $|G|^{k}$, where $k$ denotes the nilpotency index of $J(R)$. Then $R$ is a Jacobson ring for $G$.

Proof. Let $R$ be an artinian local ring. Then, $R / J(R)$ is a division ring. We shall show that $|G| \in \mathcal{U}(R / J(R))$. If $|G| \notin \mathcal{U}(R / J(R))$, then $|G| \in J(R)$. Since $k$ denotes the nilpotency index of $J(R)$, we have that $|G|^{k}=0$. This implies $\left.m|| G\right|^{k}$.

Notice that if $m$ is prime number, we can re-write Corollary 3.3 as follows:

Corollary 3.4. Let $R$ be an artinian local ring and $G$ be a finite group such that $m=\operatorname{char}(R)$ prime does not divide $|G|$. Then $R$ is a Jacobson ring for $G$.

The next result shows that we can give a precise description of the socle of $R G$ if $R$ is a Jacobson ring for $G$.

Proposition 3.5. Let $G$ be a finite group and $R$ be a Jacobson ring for G. Then, the following equality holds:

$$
\operatorname{soc}(R G)=\operatorname{soc}(R) G=\left\{\sum_{g \in G} a_{g} g \mid a_{g} \in \operatorname{soc}(R)\right\} .
$$


Proof. Since, by hypothesis, $R$ is a Jacobson ring for $G$, we have $J(R G)=J(R) G$. Let $x$ be an element of $\operatorname{soc}(R G)$, so by Lemma 2.1, $\alpha \cdot x=0$ for all $\alpha \in J(R G)=J(R) G$. Write $x=\sum_{g \in G} x_{g} g$, then $r \cdot x=$ $\sum_{g \in G}\left(r \cdot x_{g}\right) g=0$ for all $r \in J(R)$ and for all $g \in G$. So $r \cdot x_{g}=0$ and then $x_{g} \in \operatorname{soc}(R)$. This implies that $\operatorname{soc}(R G) \subseteq \operatorname{soc}(R) G$.

On the other hand, if $x=\sum_{g \in G} x_{g} g$ with $x_{g} \in \operatorname{soc}(R) G$ and if $y=\sum_{g \in G} y_{g} g$ with $y_{g} \in J(R) G$, then $y \cdot x=\sum_{g, h \in G}\left(y_{g} \cdot x_{h}\right) g h=0$ so $x \in \operatorname{soc}(R G)$ by Lemma 2.1 .

Consequently, we have $\operatorname{soc}(R G)=\operatorname{soc}(R) G$.

Now we are ready to prove the main result of this paper.

Theorem 3.6. Let $G$ be a finite group and $R$ be an artinian ring. If $R$ is Frobenius and a Jacobson ring for $G$, then $R G$ is Frobenius.

Proof. First of all, it is not difficult to see that the set $(R / J(R)) G$ is a $R G$-module with the following multiplication $\sum_{g \in G} a_{g} g \cdot \sum_{g \in G} \overline{b_{g}} g:=$ $\sum_{g, h \in G} \overline{a_{g} b_{h}} g h$

Claim 1. $\operatorname{soc}\left({ }_{R} R\right) G \cong(R / J(R)) G$ as $R G$-modules.

Proof. Since $R$ is Frobenius, there exists an isomorphism of $R$-modules

$$
\varphi: \operatorname{soc}\left({ }_{R} R\right) \rightarrow R / J(R)
$$

and its linear extension $\tilde{\varphi}: \operatorname{soc}\left({ }_{R} R\right) G \rightarrow(R / J(R)) G$ is an isomorphism of $R G$-modules and the proof of Claim 1 is completed. 
Claim 2. $(R / J(R)) G \cong R G / J(R) G$ as $R G$-modules.

Proof. The following mapping $\phi: R G / J(R) G \rightarrow(R / J(R)) G$ given by $\phi\left(\sum_{g \in G} \overline{a_{g} g}\right)=\sum_{g \in G} \overline{a_{g}} g$ is the desired isomorphism.

Finally, since $R$ is Frobenius, we have the following isomorphism of $R G$-modules: $\operatorname{soc}\left({ }_{R G} R G\right)=\operatorname{soc}(R) G \cong(R / J(R)) G \cong R G / J(R) G=R G / J(R G)$.

Corollary 3.7. Let $R$ be a commutative artinian ring with $\operatorname{char}(R)=0$ and let $G$ be a finite group. The following conditions are equivalent:

(i) $R$ is Frobenius;

(ii) $R G$ is Frobenius.

Proof. Let $R$ be an artinian ring with $\operatorname{char}(R)=0$. By Corollary 3.3, $R$ is a Jacobson ring for $G$ so, if $R$ is Frobenius, by Theorem 3.6, $R G$ is Frobenius.

On the other hand, if $R G$ is Frobenius, then $R G$ is $Q F$ so $R$ is also $Q F$ and, by Lemma 2.9, $R$ is Frobenius.

Corollary 3.8. Let $R$ be a commutative artinian ring with $\operatorname{char}(R)=0$ and let $G$ be a finite group. The following conditions are equivalent:

(i) $R G$ is $Q F$;

(ii) $R G$ is Frobenius.

Proof. Suppose that $R G$ is Frobenius. Then, by definition, $R G$ is $Q F$. Now, if $R G$ is $Q F$, then $R$ is $Q F$ and, again by Lemma 2.9, $R$ is Frobenius. Thus, by Theorem 3.6, $R G$ is Frobenius. 


\section{References}

[1] T. Y. Lam, Lectures on Modules and Rings, Springer-Verlag, New York, 1998.

[2] C. Polcino Milies and S. Sehgal, An Introduction to Group Rings, Kluwer Academic Publishers, Dordrecht, Boston, London, 2001.

[3] T. Nakayama, On Frobeniusean algebras I, Annals of Mathematics 40(3) (1939), 611-633.

DOI: https://doi.org/10.2307/1968946

[4] J. A. Wood, Duality for modules over finite rings and applications to coding theory, American Journal of Mathematics 121(3) (1999), 555-575.

DOI: https://doi.org/10.1353/ajm.1999.0024

[5] J. A. Wood, Code equivalence characterizes finite Frobenius rings, Proceedings of the American Mathematical Society 136(2) (2008), 699-706.

DOI: https://doi.org/10.1090/S0002-9939-07-09164-2 